\title{
A Systematic Review of Ontology-Based River Streamflow and Flood Data Management Challenges
}

\author{
Muhammad Hussain Mughal ${ }^{1,2^{*}}$, Zubair Ahmed Shaikh ${ }^{1}$, Zahid Hussain Khand ${ }^{2}$, Asif Rajput ${ }^{2}$, Faheem \\ Akhtar $^{2}$ \\ ${ }^{1}$ Department of Computer Science, Mohammad Ali Jinnah University, Karachi, Pakistan. \\ ${ }^{2}$ Department of Computer Science, Sukkur IBA University, Sukkur, Pakistan. \\ ${ }^{*}$ Corresponding author: muhammad.hussain@iba-suk.edu.pk
}

\begin{abstract}
The management of the riverine water has always remained an open challenge. The variation of water flow creates hurdles to determine the exact time and the quantity of water flow caused by the spatio-temporal complex streamflow and flood risk reduction domain. From a management perspective, irregular flow patterns generate various challenges and the development of irrigation water distribution schemes without contextual knowledge integration adversely affect the relevant community. The river streamflow and flood mitigation domains are interdisciplinary that require coordination from the various stakeholders. Coordination limiting factors includes native data acquisition methodology of each stakeholder for their specific needs, the complexity of the domain involving a heterogeneous group of managers, spatio-temporal context, region-specific terminologies, data sharing, and reusability support. Earlier proposed research and developed ontologies by the esteemed researchers focused to address these challenges in a domain-specific context. In this research, we review the challenges of a large scale spatio-temporal system for streamflow of watersheds and flood disaster management based on the ontological semantic models. This research also examines the proposed ontological models for streamflow and/or flood domain, and how they address such challenges. Furthermore, a systematic review of the last two decades' research articles is conducted and the findings are presented to assess the mappings of the challenges to proposed solutions through ontological modeling for streamflow and flood domain.
\end{abstract}

Keywords-Semantic model, interoperability, information visualization, inference, explicit information, conceptualization, ontology, flood, streamflow

\section{Introduction}

$\mathrm{F}$ LOOD disaster affects an agricultural country's communities by casualties, injuries, infrastructure damages, livelihood directly and economy of the country indirectly depending on the magnitude of the flood. Proactive measures for prevention and mitigation enriched with contextual knowledge contribute effectively to avoid such disastrous situations. For developing countries, a flood is one of the most frequent and devastating disaster, especially for agricultural countries having complex irrigation networks with spatio-temporal streamflow variation and managed by the various administrative authorities with a different mandate. The streamflow data acquisition techniques

ISSN: 2523-0379 (Online), ISSN: 1605-8607 (Print)

DOI: https://doi.org/10.52584/QRJ.1901.06

This is an open access article published by Quaid-e-Awam University of Engineering Science $\&$ Technology, Nawabshah, Pakistan under CC BY 4.0 International License. at different locations with different magnitudes are managed by the distributed authorities. A flood management system usually requires an integrated and inter-operable information representation portal for a community-centric watershed with river characteristics, rainfall conditions, stream-flow data, community location, accessibility to a safe location, effective coordination mechanism among response providers, and disaster managing organization. One major challenge of flood disaster is the coordination among stakeholders for pre-, during, and post-flood disaster activities to mitigate its adverse effects. The coordination challenge demands a common conceptualization of the streamflow for understanding, modularization, extendibility, accessibility, and reusability of integrated flood information as reported by Agresta et. al[1].

The ontology models based on the information extracted from the correlating domains provides even 
better results by integrating reusable ontologies of the SWEET suit, especially for the forest, vegetation, weather, and geographic domain to cover fire[2]. Our research emphasize on technical studies on the use of an ontology-based system for water governance [3], flood risk assessment[4], flood information management [5], indexed in academic databases for semantic modeling. However, limited research is published as a review article on the ontological approach for flood [6], for instance, Rina Suryani Oktari et. al. [7] presented a review on knowledge management practice for disaster management generally based on people, process, technology, and goal from single Scopus database and knowledge management for generic disasters. However, we focus on knowledge management and representation for streamflow optimization and flood mitigation aspects.

Another flood ontology systematic review was conducted by Sinha and Dutta [6] with parametric approaches and objectives to extract common concepts in existing flood ontologies, key parameters, and gaps. The review represented by the flood ontological models with parameters of the creator, sponsoring organization, purpose, types, focus phases, reusability support, domain, and design methodology. The developed ontologies are assessed based on class hierarchy, levels, representation language, formality levels, IDEs used for development, evaluation, library, compliance, etc. However, our review focus is to rationalize the need for a knowledge-base system not only for a flood, but also streamflow data acquisition, management, representation, and dissemination aspects.

To overcome the research gaps of the aforementioned review articles and limitations. We cover computational challenges for implementation perspective and sustainability of the large-scale systems the ontology-based systems for challenges related to the large-scale system with multiple data sources and coordinating organizations. More specifically, this review will attain the following main objectives.

1) A detailed review of research studies, proposals, and implemented ontologies forriver streamflow, and flood management domain.

2) To investigate the challenges of river streamflow and flood management domain, especially, the large-scale system.

3) To comparatively assess these ontologies against potential challenges.

4) To investigate the challenges not addressed or poorly addressed in the existing literature.

The remainder of this paper is structured as follows. Section 2 presents the research methodology for selecting the research studies in the domain of river stream- flow and flood's data management ontologies. Section 3 presents the challenges for streamflow and flood data modeling. This section highlight spatio-temporal river streamflow relation with flood management and data modeling challenges. Section 4 presents semantic modeling features for streamflow and flood data management. Section 5 presents a detailed discussion, and Section 6 concludes the paper with research gaps and way forwards to design sustainable systems.

\section{Research Methodology}

A domain-specific systematic literature review (SLR) extracts the depth of a particular research domain and sorts out the quality of research article for a particular domain for other scientists with a holistic review of the existing publications along with progression, open dimensions, trends, active researchers, groups or individuals in that particular area. Our review methodology has three phases: literature identification, identification of streamflow and/or flood domain challenges, and asses ontological models features to cope with these challenges from a computational perspective. The first phase of research articles selection criteria has six groups. These groups are keywords of river, flood and ontology domain, publication year, type of publication, and language. These groups and final query formation is presented in Table 1 . Then article relevancy is assessed from the title of the paper, screening based on the contents of the abstract and conclusion. The inclusion/exclusion criterion is discussed in Section 2.2. The article selection process following the PRISMA [8] guideline of the review articles selection is shown in Figure 1.

\subsection{Keyword Selection}

The systematic literature review begins with identifying keywords and search terms that retrieve relevant articles from online research databases, the keywords heuristically selected for targeted studies keeping synonyms, and connected sub-domain for a broader perspective of a targeted investigation. Special care is required for keyword selections due to some words' different meanings in different disciplines, domains, and languages. The selection of research articles requires identifying domain keywords used for selecting appropriate research articles from online research databases. The sequences or patterns of terms and search strategy also affects the query results. We found the following basic search strings to be most appropriate for the river streamflow and semantic-based flood models from the research objectives and exploratory search. 


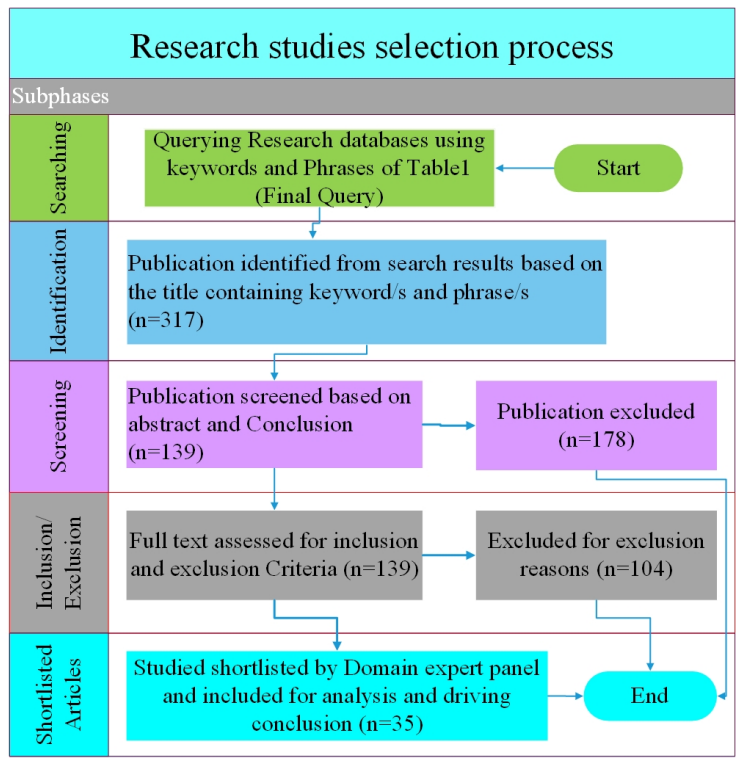

Fig. 1: Research studies selection process and phases

The keywords "river streaming", "flood ontology", "flood management", "semantic model", "flood assessment", "flood response", "ontological", "ontologybased", "irrigation system", "river flow system", and "flood mitigation" are used to form search strings. These keywords are joined using AND/OR logical operators for stick and relaxing combination. These keywords are sensibly permuted to maximize the intended selection of the domains' research studies. The detailed search criteria is shown in Table 1 . The query results were screened from the title, abstract and keywords of articles to consider for detailed review. This review includes the analysis of relevant studies searched from famous scientific research databases and search engines. The following eight electronic bibliographic databases were queried using the final search query.

1) Google Scholar

2) IEEE Xplore

3) ISI Web of Science

4) Kluwer Online

5) Wiley Interscience

6) Science Direct - Elsevier

7) Springer Link

8) Semantic Scholar

\subsection{Inclusion/Exclusion Criteria of Research Studies}

In this paper, we included the research studies proposing, recommending, designing, developing, discussing the challenges of environmental models generally and river streamflow models specifically using ontologies or semantically enriched models. Similarly, we investigated the studies of environmental disasters in general and ontology-based riverine flood management, mitigation, response, and prevention in a specific con-text.

We have excluded ontological studies emphasizing disaster other than flood domain or discussing water quality or water in pipelines and household supply. Our keen interest was in the investigation of streamflow management connected with flood avoidance and optimized freshwater distribution through an irrigation network. The articles using search words, but in other contexts or domains; for example, the article contains the words "Flood" and "Ontology" but in the network, traffic floods are not relevant to this review. Lastly, the irrelevant studies based on the title, abstract, conclusion, and full paper review, screened iteratively and removed from the final selection list. We included the studies on surface freshwater waterways and analyzes the impact of flood disasters [7] originating from these freshwater waterways and irrigation networks.

\section{Challenges for Streamflow and Flood Data Modeling}

The riverine flood jerk down the prosperity rate and is a frequent disaster for agricultural countries, having spatially distributed large scale rivers, canals, channels, and watercourse network for irrigation. The monsoon season normally becomes alarming due to the variable melting rate of glaciers and irregular patterns of heavy rainfall, climate change [9], and an increase in temperature and environmental pollution from increasing industrialization without inadequate proactive measures. The increasing scarcity of water demands for optimization of water resources through the use of drip irrigation [10] based systems and distribution of water for crops needs. The a dire need for the designing and development of a sustainable framework and data-intensive decision support system by coping domain challenges. The streamflow and flood domain challenges includes heterogeneous data sources of streamflow estimation such as remote sensing [11], wireless sensor network[9], normalization of domain complexity, the variability of spatio-temporal context, the incompleteness of the data, expressiveness of the models, automated information retrieval, visualization of large scale irrigation data, and scalability of system.

\subsection{Data Sharing}

Due to the interdisciplinary model across correlated domains, data sharing is a basic need for collaborative decision support systems. Every organization 


\begin{tabular}{|c|c|}
\hline Group-1: River stream flow-related keywords & $\begin{array}{l}\text { Irrigation system, river flow, River Management, Breach detection, } \\
\text { river diversion, flow measurement }\end{array}$ \\
\hline Group-2: Flood related keywords & $\begin{array}{l}\text { Flood risk reduction, Flood Management, Flood Response, flood mitigation, } \\
\text { Flood Assessment, inundation, inundated areas, swamp, deluge, torrent, } \\
\text { River overflow, freshet }\end{array}$ \\
\hline Group-3: Semantic-based keywords & $\begin{array}{l}\text { Ontology, Semantic, ontological, Semantic, Knowledge base, } \\
\text { Knowledge Management }\end{array}$ \\
\hline Group-4: Publication year & January 2005 to October 2020 \\
\hline Group-5: Document type & Journal or Conference Articles \\
\hline Group-6: Language & English \\
\hline Final Search Query & $\begin{array}{l}\text { ( } \exists \text { C1 } \in \text { Group-1)AND }(\exists \text { C2 } \in \text { Group-2)AND }(\exists \text { C3 } \in \text { Group-3) } \\
\text { AND }(\forall \text { Group-4) AND }(\forall \text { C4 } \in \text { Group-5) AND (Group-6) }\end{array}$ \\
\hline
\end{tabular}

TABLE 1: Grouping research article searching criteria

maintains their data repositories and use their native format that suits organization's needs. Defining uniform and standard data sharing mechanics is an open challenge for such systems. The emergence of data sharing in-creases, especially in a crisis management scenario at the time of flooding [12]. The ontology curate and structure the domain data for effective sharing among the stakeholders and coordinating citizen participators. Ontologies are effective tools for domain data retrieval, discovery, and sharing in emergency and crisis management [22]. The streamflow and flood management domain data is usually in a spatial form and need to be shared across the range of stakeholders to process the spatial data of different resolution from various remote sensing techniques and geographical information systems [30]. Khantong et. al. in [13], discussed ontology-based disaster response in emergency management and its effectiveness for stakeholders coordination.

\subsection{Heterogeneous Data Sources}

Efficient flood forecasting does not only require the adequate spatio-temporal context of rivers or canals network. The context includes hydraulic, hydrologic, meteorological, environmental data along with inclination of river passage, and embankment condition[14] using remote sensing technique. The data scarcity at any point in the network raise uncertainty to model flood mitigation strategy. To fill the gap of missing data, multiple source integration is needed. For the sake of example, zhu et. al. [16] used approaches to acquire weather data from citizens through crowdsourcing integrated with the actual sensor data to optimize the ac-curacy and availability of data but raise integration challenge for heterogeneous sources. Flood situations and inundation data acquired from remote sensing techniques are integrated with citizen observation for flood risk assessment [17]. The geophysics data obtained by crowdsourcing [18], remote sensing, and survey techniques are heterogeneous data sources. The flood and streamflow information is shared through social media by the affected community [15]. Provenance information of the distributed water resource management systems, acquiring the data from heterogeneous sources of the integrated systems im-proved the quality of the information [13].

In [19], rainfall and runoff data may be integrated with remote sensing and wireless sensor data to improve the data quality of multiple sources and filling the missing data of one source with the other. The administrative authorities need to develop an efficient system based on integrations with social media, remote sensing, crowdsourcing, WSN data, telemetry data, manual gauging, stochastic modeling data, and other heterogeneous data sources to complement each other. There exist a need to design a cost-effective and efficient solution, where the data was not collected due to sensor fault, unavailability of gauging personnel, or any other reasons. The geo-social proxy variable [20] data integrated with official data and hydrology increased streamflow modeling accuracy from $39 \%$ to $58 \%$, and reduced uncertainty from $71 \%$ to $29 \%$ using the probability distribution model. These scenarios represent the challenges of heterogeneous data sources for design and development of an efficient and sustainable decision support system for streamflow optimization, environment monitoring, flood risk identification, and mitigation.

Grigori et. al. [16] introduce the SoKNOS support system to handle the challenge of data heterogeneity exchange among multiple organizations during a disaster response situation to improve the efficiency of the system and to enable other semantic web-based soft-ware to use the information normalized by semantic web technologies. They used Onto-Broker for the integration of the database of coordinating organizations and Joint Query Engine (JQE) for information retrieval of the heterogeneous sources. Caleb Goodwin 
et. al. developed a prototype for proof of concepts for onto-sensor, an ontology-based heterogeneous data generated from wireless senor network management system for environmental monitoring in [17]. Their prototype acquires the data from temperature, Global Positioning System(GPS), barometric pressure, and acoustic measurements relevant to the flood modeling aspects. Concluding the heterogeneous data source, the ontology-based models are an efficient way of mapping heterogeneous data to unify for query languages [18]. For an efficient forecasting system, a minimum of three heterogeneous systems hydraulic, hydrologic, and data sources for stream gauge or sensor network data required [1].

\subsection{Distributed Computing}

The streamflow and flood data acquisition, preprocessing, and management operations are distributed among regional organizations for need-based intra- and inter-communication of the data and publishing on their website without semantic information. The distributed computation and processing of streamflow in rivers, canals, watercourse, and dames is based on the local and/or organizational predefined standards. Variation of streamflow due to climate variation of rainfall and temperature needs to be communicated to other relevant authorities for computation semantics. Stream-lining these distributed computational practices are challenges of a sustainable river streamflow flood mitigation framework. The interoperating and collaborating institutions process their domain information by inter-locking their institution world [13].

\subsection{Semantics}

The large-scale systems and the processes involve large numbers of entities, and activities have different semantics in different contexts. The streamflow optimization workflow requires semantics to resolve the conflict among the multiple stakeholders for streamflow distribution in distry or link channel. Flood forecasting requires modeling the semantic heterogeneity of water-sheds, environmental data from various sensors, citizen observational data, and manual gauging by irrigation personnel. Scientific communityrecommended ontology based solutions for semantic support towards design, development, and implementation of the complex integration of multiple domains with heterogeneous data [1].

\subsection{Spatio-Temporal Variability}

There is a strong correlation between the flood impact and spatio-temporal variation of hydrological extreme conditions. We believe that efficient and sustain-able systems cannot be developed without considering the spatio-temporal variability of precipitation, rainfall, runoff, water level effects due to rainfall, temperature, humidity, precipitation, and soil condition. Capturing the spatio-temporal variability impacts of climate change would be a valuable effort for flood risk reduction improvement [9]. The spatio-temporal hydrological data needs a mapping for hydrological flood assessments, this assists to tackle the high complexity of the flooding and disaster possible impacts. Modeling the spatio-temporal variations of the hydrological extremes conditions would use for future smart systems.

\subsection{Data Incompleteness}

We believe that a single streamflow measurement technique not capable to acquire the complete stream-flow data. Various techniques are used for data acquisition each has some tradeoffs. The manual gauging of the streamflow is laborious and low data generation frequency, remote sensing provides daily or weekly im-ages but requires extensive image processing, sensor network provides data with high frequency but may be noisy, due to malfunctioning of the sensor. No single technique can provide complete data with high frequency, max quality, minimum processing, and maintenance overheads

\subsection{Information Representation}

The tabular and/or file-based representation of the complex irrigation, streamflow measurements, and flood mitigation experts may misinterpret the information. A large-scale system involving various interconnected domains has concepts with different interoperations depending on the domain and context requires an efficient means of information representations. The graphical information representation of explicitly defined entities with multiple relation and interaction contexts is considered better information representation techniques than tabular, textual, and numerical data without semantics. The ontology-based models are useful for handling heterogeneous data semantics and preserving the data semantic and information representation for humans and machines alike. The flood forecasting model efficiency depends on audience-based information representation and coping with heterogeneous information representation semantic $[1]$.

\subsection{Complexity}

The proactive flood risk reduction strategies formation based on the efficient utilization of irrigation network 
context reduce to probability floods. The distribution of water resource by integration with agricultural, hydraulics, hydrology, meteorological, infrastructure condition, capability assessment, vulnerable communities, equipment for rescue and response, reliability of information sources, coordination among stakeholders raise the complexity of the system. The complexity challenge requires explicit annotation of the concepts enables to normalize streamflow and mitigation process.

We review the hydraulic, streamflow, surface water, and flood management ontology representing research articles to deduce their ontology-based systems analysis. Yusuf Sermet et. al. [5] highlighted the need for information-centric flood ontology to cope with challenges of scalability of large scale environmental, spatial, and collected from various heterogeneous sources, lacking structure. To handle the emergency of a meteorological disaster, S. Zhong et. al. [19] proposed geo-ontology for semantic reasoning of complex domain acquiring heterogeneous data sources. They discussed the level-based approach for top, domain, task, and application ontologies and their purposes. They also highlighted the need for spatio-temporal context extraction for emergencies and disaster management. The proposed do-main and task ontologies for disasters covering a spatio-temporal context. The modular approach for subontologies such as Disaster Meteorological Event(DME), emergency management, process ontology, and so on resolves complexity challenge for connecting different interrelated domains in the module with concepts required for specific objective.

Zening et. al. [29] discussed the complexity of heterogeneous data management of urban flood disasters through ontology development. The data collected from heterogeneous sources such as internet data, sensor data, social data, and statistical data in different formats, resolution, language for rainfall, urban environment needs to normalize in standard form for computation and recommendation for adequate proactive, during, and post-disaster actions.

Towards the provision of normalized data from heterogeneous sources of sensors, databases, maps, and other sources for web-based decision support applications was to manage flood situation on the sea site was discussed by Gray et. al. in [20]. The data of heterogeneous sources need data sharing for application and humans with improved visualization that perceiving them as data from a single source. The proposed sensor web semantic for emergency response planning. Elag and Goodall define a component-based ontology model for water resource management [21]. They proposed to re-use the existing ontology of Semantic Web for Earth and Environmental Terminology (SWEET) modular and integrated it with Water Resource Component Ontology (WRCO). They concluded that the need for an ontology for water resource management due to multidisciplinary, community-focused, interoperable, component-based interconnected domains model for knowledge level and join conceptualization of the water resource model. Khantong et. al. [13] described a modular ap-proach for managing the disaster response and data sharing ontology.

Table 2 highlights the research studies dis-cussed, asserted or mentioned challenges for stream-flow and flood data modeling. The data acquired from hydrological sensors are heterogeneous, an ontology-based solution of SSN ontology integration with integrated with time and space ontology capturing Spatio-temporal context developed flood ontology presented in [22].

\section{Semantic Modeling Features for Stream- flow and Flood Data Modeling}

Bermudez et. al. [23] preferred to use the ontologybased modeling for the hydrological domain for data sharing among communities to tackle the difficulties of increased data volume, variety of data measuring techniques, context-specific structure, and heterogeneity of information systems. Alsayed et. al. leveraging the ontological model [24] through modularization techniques. The multidisciplinary ontology especially having a high granularity of concept and relation, needs to be compartmentalized for the efficiency of specific users working on the portion of ontology. An ontology partitioning technique developed and implemented that analyzes the seed for partitioning is named as Ontology Analysis and Partitioning Tool(OAPT).

\subsection{Inter-operability}

The data is a key to develop efficient decision support systems but the generation of data by different sources raised the issue of inter-operability for a decision support system, especially during an emergency. Re-searchers adapted the ontology-based system for inter-operability challenge as presented in [12] by Roller, R., J. Roes, and E. Verbree. The adaption and utilization of the ontology across multi-platforms resolved the inter-operability challenge and this feature was supported by the semantic web technologies through the use of standardized inter-platform supporting language such XML(eXtensible Markup Language), RDF(Resource Description Framework), OWL(Ontology Web Language). The integration of the various correlated domain data and systems has 
the challenge of inter-operability for topographic and flood defense ontology presented by Dolbear et. al. in [27]. The IKNOW model proposed by Liu et. al., in [26] provides inter-operability among heterogeneous systems. Fang et. al. [9] presented the requirement of an inter-operable ontological system leveraging the semantic data from various correlated do-mains enabled a reduction of losses magnitude in dis-aster management systems.

\subsection{Modularization}

Primarily, there are two system partition reasons, logic base and structure base. A modular approach preferred over a tightly coupled component of large systems provides easy debugging, maintainability, expandability, scalability, and prevention from a single point of failure. The semantic model has an inherited benefit of the modular approach, especially for a disaster management systems, where the failure of the data management, representation, and communication increases the mortality, financial, economic, demographical, and social losses. The ontology-based modular and semantically enhanced systems are required for disaster management, risk reduction [28]. WaterOnto [29] modular ontological-based water management system for irrigation water management and flood reduction incorporating the contextual information from the grid of the wireless sensor network. The Grid-based WSN [30] for flow monitoring and river breach identification considering the environmental, management constraints along with other contextual information.

\subsection{Integration}

The solo models perform relatively unsatisfactory and ineffective concerning the large-scale and especially for interconnected domains. The integration of multiple systems and models contributes many folds towards the achievement of objectives and has a more acceptability rate user for efficiency and efficacy. The flood risk assessment model needs integration with interrelated domains. The case study of Cedar River Basin under the property Buyout project used an integrated approach for benefit-cost analysis and flood risk analysis of the property. They consider that streamflow, flood, and environmental conditions for potential property buyout cost benefits and avoid the possible damages in the State of Iowa [31]. Qing et. al. in [26] integrated the ontologies representing the distributed water model and provenance information of the heterogeneous water re-sources and information system named "IKNOW", and also evaluated this model from the two real-world water information systems. The ontology-based integration of wireless sensors network is proved through prototype development for the environment modeling in [12]. Flood risk assessment prioritizing and integrating the stakeholder's preference which is normally ignored proved the efficacy of the integration [22]. The sustainable models for integration of watershed digital data of spatial data sources and GIS tools for integrated assessment ontologies are presented in [32].

\subsection{Semantic Reasoning}

The semantic model provides tools for the semantics of the data and the capability to derive the knowledge using logical reasoning from existing concepts. The power of semantic reasoning is based on the expressivity of the domain information embedded through explicit annotations of the domain data. The domain information is backed by Description Logic [33] derived from First Order Logic enables to infer the tacit knowledge from explicit annotation. A variety of semantic reasoner exists to derive the knowledge such as rule-based languages [34]. Semantic web language offers various levels for inference and logic embedding knowledge instead of logic in application and knowledge model separately. The XML is the lowest level of semantic language. The RDF (Resource Description Framework) and RDFS (RDF Schema) comparatively have more attribute level description and data types for additional schematic level. The OWL (Ontology Web Language) version 1.0 and 2.0 with highlevel support for expression through properties. All semantic languages are bottom-up compatible. Various semantic rule languages provide logical inferencing support such as FOL-RuleML (First Order Logic Rule-Markup Language, SWRL(Semantic Web Rule Language), Natation3, Jena Rule, and RIF(Rule Interchange Format). FOL-RuleML) is the sub-language of Rule-ML that sup-ports binary logical and biimplication inferencing of semantic model. SWRLin addition to RuleML provides many built-in functions support for RDF, RDFS, and OWL. In 2008, Notation3 was submitted by W3C to ex-tend RDF by rule adding formulae and logical implementation. RIF [35] is a W3C recommendation to ex-change rules among disparate systems. FOL-RuleML, SWRL, and RIF use XML for interchange format and support compatibility requirements. SWRL enables adding rules for further formal and explicitly describing the semantic of the domain. Various reasoner Fact++, HermiT, Pellet [34], ELK, jcel, Racer assure the consistency of OWL. HydrOWL and other support inferred knowledge from 


\begin{tabular}{|c|c|c|c|c|c|c|c|}
\hline Research Study & 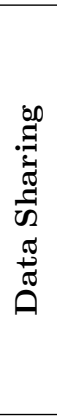 & 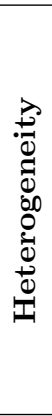 & 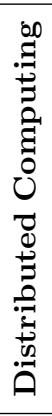 & 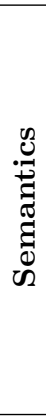 & 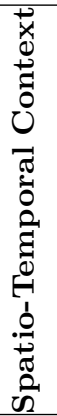 & 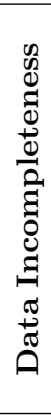 & 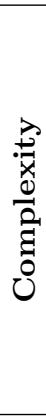 \\
\hline Information-centric flood ontology $[5]$ & $\mathrm{X}$ & $\mathrm{X}$ & $\mathrm{X}$ & & & & \\
\hline Geo-ontology-based emergency management [10] & & $\mathrm{X}$ & $\mathrm{X}$ & $\mathrm{X}$ & $\mathrm{X}$ & $\mathrm{X}$ & \\
\hline Ontology-based framework for flood disasters [11] & $\mathrm{X}$ & $\mathrm{X}$ & $\mathrm{X}$ & $\mathrm{X}$ & $\mathrm{X}$ & & \\
\hline A semantic sensor web for environmental Decision Support [12] & $\mathrm{X}$ & $\mathrm{X}$ & $\mathrm{X}$ & $\mathrm{X}$ & $\mathrm{X}$ & & \\
\hline Ontology for Sharing and Managing Information in Disaster Response [13] & $\mathrm{X}$ & $\mathrm{X}$ & $\mathrm{X}$ & & & & \\
\hline SoKNOS $[14]$ & $\mathrm{X}$ & & & $\mathrm{X}$ & & & $\mathrm{X}$ \\
\hline Ontology-based knowledge management framework for a distributed water [15] & $\mathrm{X}$ & $\mathrm{X}$ & $\mathrm{X}$ & $\mathrm{X}$ & & & \\
\hline framework for flooding forecasting [1] & & $\mathrm{X}$ & & & & & \\
\hline SSN Ontology $[16]$ & & $\mathrm{X}$ & & $\mathrm{X}$ & $\mathrm{X}$ & & \\
\hline Linked data for crisis management [17] & $\mathrm{X}$ & $\mathrm{X}$ & $\mathrm{X}$ & $\mathrm{X}$ & & & $\mathrm{X}$ \\
\hline
\end{tabular}

TABLE 2: Challenges for streamflow and flood data modeling

explicit classes and relations. The famous ontology development IDE protégé and Jena Framework provide support for these reasoners. The HermiT is builtin reasoner in protégé 5.5 .

\subsection{Visualization}

A famous English Language adage "A picture is worth a thousand words" represents the importance of powerful features of semantics and ontology-based model. Graphically, visualizing the entities, relation, assertion, and constraints of a domain ontology are means to simplify the complexity of the large set of entities with multiple interactions, and even the interaction having symmetric, reflexive, anti-symmetric, irreflexive, and transitive properties. This linked representing different semantic through graph data structure with different color coding for each interaction enhances the understanding of a human about some domain the easiest way compared to text and numerical data. With continuous improvements in $5 \mathrm{G}$ cellular networks [36] the visualization of images and graph data challenges are resolved. The information about flood disaster visu-alization is a key challenge for decades [37]. The building tools and addon for RDF and OWL visualization include Owlvis, ontoGraph, allegro, and other graph data based storage and visualization [38].

\subsection{Extendability}

An existing ontology can be extended or reused in another system that uses ontology-based knowledge representation system. Adding the concepts, relations, and properties of the classes in existing ontology is known as ontology expandability. Flood domain ontology is extended for information needs and regions specific encoding for local terminologies [5]. Developing an ontology and reusing concepts defined in existing ontology fully or partially known as reusability is discussed in the following section. Ontologies are designed for easy maintainable, extendable structures with minimum effort [39]. Expendability and maintainability of ontology and preserving semantic are supported by ontological modeling [40].

\subsection{Reusability}

The reusability is yet another advantage of the ontological based models. The domain ontologies formally conceptualize entities of the domain capturing the context of the extract with other entities of the same and other relevant domains' development by other organizations their domain of interest. These techniques enable the integration of the correlating domain to provide even better results from reusing existing ontologies. A famous Semantic Web for Environment and Earth Terminologies (SWEET) ontology leverages the knowledge of earth science such as forest, vegetation, weather, geo-graphic domain, and related disasters cover fire [2] do-main, and flood Management domain [41]. Similarly, Environmental Impact Assessment (EIA) ontology for flood management [41], SWEET, and others for decision support based on semantic sensors [13] are examples of the reusability of ontologies. 
Although there is a challenge faced by ontologists matching and merging different ontologies.the ontology editing software support for these challenges to integrate ontologies of different broader domains such as medicine, geography, and agriculture. The comprehensive survey covering content-based and contextbased ontology matching techniques from 2003 to 2013 was explained in the review article [42].

Julián Garrido et. al. developed Flood Ontology [43] from the recommendation of METHONTOLOGY an Ontology development methodology reusing the EIA ontology leveraging most of the flood-related already defined concept in EIA ontology. The reusability characteristic of ontology-based modeling motivated the ontologist to partition ontologies into a domain, task, and application ontology. The domain ontologies are integrated and extended for task ontology to accomplish the task of some specific module.

\section{Discussion}

This review paper covers the various approaches proposed by esteemed researchers for river data acquisition, processing, management, and representation using ontological models. The highlights of this review concluded that domain having variation of information based spatio-temporal context, concerning with heterogeneous group of stakeholders, data sources, representation and management systems.Ignorance of variation leads to a disaster like floods. These challenges need to be addressed with semantic web technologies for ma-chine-understandable semantics towards automation of laborious information acquisition and dissemination.Reducing the ambiguity of data, and interpretation generate obstacles for optimization. The research direction for context-oriented irrigation semantic modeling is open.

\section{Conclusion \& Future Direction}

This paper reviewed existing research to extract the challenges that triggered the river streamflow and flood management domain towards ontology-based solutions. The ontology-based semantic flood modeling systems provide inter-operability of heterogeneous data sources in various organizations using different plat-forms and the modular approach model for distributed but logically connected computing components to ex-change the data for data-intensive decision support and recommendation systems. Semantic reasoning to derive new knowledge from explicitly defined entities and relations. The reusability feature contributes to cost-effectiveness and time-saving rather than developing a system from scratch. The easy integration to connect the relevant domain for computation, storage, and data ex-change. Graphical visualization of the complex domain is easily understandable by humans and annotated da-ta with semantic information is suitable for machine processing. The ontology-based systems are easily extendable, which focus on solving scalability related is-sues. In the future, a framework needs to be developed, mapping heterogeneous data sources of remote sensing, manual gauging, wireless sensors, crowdsourcing for irrigation domain-specific rule inferencing, and flood mitigation considering spatio-temporal context and variation of streamflow in the irrigation domain. The blockchainbased consensus development among stakeholders to resolve inter and intra states or countries administration's conflict on river streamflow distribution as an extension of semantics framework data and decision's reliability.

\section{References}

[1] A. Agresta, G. Fattoruso, M. Pollino, F. Pasanisi, C. Tebano, S. De Vito, et al., "An ontology framework for flooding forecasting," in International Conference on Computational Science and Its Applications, pp. 417-428, 2014.

[2] K. Kalabokidis, N. Athanasis, and M. Vaitis, "OntoFire: an ontology-based geo-portal for wildfires," Natural Hazards and Earth System Sciences, vol. 11, p. 3157, 2011.

[3] S. Campbell and L. Gurney, "Mapping and navigating ontologies in water governance: the case of the Ganges," Water International, pp. 1-18-1-18, 2020.

[4] S. Yi and Y. Xiao, "The development of integrated watershed flood risk assessment ontology," in 2015 23rd International Conference on Geoinformatics, pp. 1-6, 2015.

[5] Y. Sermet and I. Demir, "Towards an information centric flood ontology for information management and communication," Earth Science Informatics, vol. 12, pp. 541-551, 2019.

[6] P. K. Sinha and B. Dutta, "A Systematic Analysis of Flood Ontologies: A Parametric Approach," KO KNOWLEDGE ORGANIZATION, vol. 47, pp. 138-159-138-159, 2020.

[7] P. Punnarak, P. Sojisuporn, H. Jitrapat, and A. Piumsomboon, "Effect of Flood Disaster and Long Term Changes during 2011 to 2018 on Community Structure of Zooplankton in the Inner Gulf of Thailand," International Journal of Environmental Science and Development, vol. 11, 2020.

[8] A. A. Selçuk, "A guide for systematic reviews: PRIS-MA," Turkish archives of otorhinolaryngology, vol. 57, p. 57, 2019

[9] J. Fang, F. Kong, J. Fang, and L. Zhao, "Observed changes in hydrological extremes and flood disaster in Yangtze River Basin: spatial-temporal variability and climate change impacts," Natural Hazards, vol. 93, pp. 89-107-89-107, 2018.

[10] W. M. A. Khalifa, H. Gasmi, and T. A. Butt, "Farm-Based Environmental and Economic Impacts of the Drip Irrigation System," Engineering, Technology \& Applied Science Research, vol. 10, pp. 6335-6343, 10/26 2020.

[11] D. M. Bjerklie, C. M. Birkett, J. W. Jones, C. Carabajal, J. A. Rover, J. W. Fulton, et. al., "Satellite remote sensing estimation of river discharge: Application to the Yukon River Alaska," Journal of hydrology, vol. 561, pp. 10001018, 2018. 


\begin{tabular}{|c|c|c|c|c|c|c|c|}
\hline Research Study & 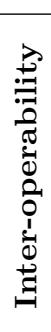 & 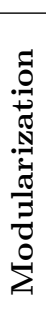 & 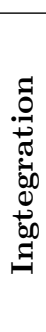 & 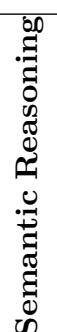 & 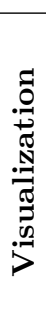 & 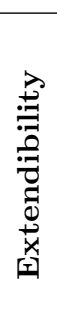 & 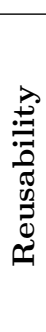 \\
\hline Information-centric [5] & $\mathrm{X}$ & $\mathrm{X}$ & $\mathrm{X}$ & & $\mathrm{X}$ & $\mathrm{X}$ & \\
\hline Emergency management [10] & & $\mathrm{X}$ & $\mathrm{X}$ & $\mathrm{X}$ & $\mathrm{X}$ & & \\
\hline Framework for flood disasters [11] & $\mathrm{X}$ & $\mathrm{X}$ & $\mathrm{X}$ & $\mathrm{X}$ & $\mathrm{X}$ & & \\
\hline Environmental Decision Support [12] & $\mathrm{X}$ & $\mathrm{X}$ & $\mathrm{X}$ & $\mathrm{X}$ & $\mathrm{X}$ & & \\
\hline Sharing and Managing Information in Disaster Response [13] & $\mathrm{X}$ & $\mathrm{X}$ & & & $\mathrm{X}$ & $\mathrm{X}$ & $\mathrm{X}$ \\
\hline SoKNOS $[14]$ & & & $\mathrm{X}$ & $\mathrm{X}$ & $\mathrm{X}$ & $\mathrm{X}$ & $\mathrm{X}$ \\
\hline A knowledge management framework for a distributed water [15] & $\mathrm{X}$ & $\mathrm{X}$ & $\mathrm{X}$ & $\mathrm{X}$ & & & $\mathrm{X}$ \\
\hline Flooding forecasting framework [1] & $\mathrm{X}$ & $\mathrm{X}$ & $\mathrm{X}$ & & & & $\mathrm{X}$ \\
\hline SSN Ontology $[16]$ & $\mathrm{X}$ & & $\mathrm{X}$ & & & & \\
\hline Linked data for crisis management [17] & $\mathrm{X}$ & $\mathrm{X}$ & $\mathrm{X}$ & $\mathrm{X}$ & & & \\
\hline WaterOnto [18] & $\mathrm{X}$ & $\mathrm{X}$ & $\mathrm{X}$ & & $\mathrm{X}$ & $\mathrm{X}$ & $\mathrm{X}$ \\
\hline
\end{tabular}

TABLE 3: Ontology-based semantic model features

[12] R. Roller, J. Roes, and E. Verbree, "Benefits of linked data for inter-operability during crisis management," The International Archives of Photogrammetry, Remote Sensing and Spatial Information Sciences, vol. 40, pp. 211-211, 2015.

[13] S. Khantong and M. N. Ahmad, "An Ontology for Sharing and Managing Information in Disaster Response: In Flood Response Usage Scenarios," Journal on Data Semantics, pp. 1-14-1-14, 2019.

[14] M. V. Japitana, A. T. Demetillo, M. E. C. Burce, and E. B. Taboada, "Catchment characterization to support water monitoring and management decisions using remote sensing," Sustainable Environment Research, vol. 29, pp. 110, 2019.

[15] C. Restrepo-Estrada, S. C. de Andrade, N. Abe, M. C. Fava, E. M. Mendiondo, and J. P. de Albuquerque, "Geosocial media as a proxy for hydrometeorological data for streamflow estimation and to improve flood monitoring," Computers \& Geo-sciences, vol. 111, pp. 148-158, 2018.

[16] G. Babitski, S. Bergweiler, O. Grebner, D. Oberle, H. Paulheim, and F. Probst, "SoKNOS-using semantic technologies in disaster management software," in Extended Semantic Web Conference, pp. 183-197, 2011.

[17] C. Goodwin and D. J. Russomanno, "An ontology-based sensor network prototype environment," in Proceedings of the Fifth International Conference on Information Processing in Sensor Networks, pp. 1-2, 2006.

[18] K. Grolinger, M. A. M. Capretz, E. Mezghani, and E. Exposito, "Knowledge as a service framework for disaster data management," in 2013 Workshops on Enabling Technologies: Infrastructure for Collaborative Enterprises, pp. 313-318, 2013.

[19] S. Zhong, Z. Fang, M. Zhu, and Q. Huang, "A geoontology-based approach to decision-making in emergency management of meteorological disasters," Natural hazards, vol. 89, pp. 531-554, 2017.

[20] A. J. Gray, J. Sadler, O. Kit, K. Kyzirakos, M. Karpathiotakis, J.-P. Calbimonte, et. al., "A semantic sensor web for environmental decision support applications," Sensors, vol. 11, pp. 8855-8887, 2011.

[21] M. Elag and J. L. Goodall, "An ontology for componentbased models of water resource systems," Water Resources
Research, vol. 49, pp. 5077-5091, 2013.

[22] C. Wang, N. Chen, W. Wang, and Z. Chen, "A hydrological sensor web ontology based on the SSN ontology: A case study for a flood," ISPRS International Journal of GeoInformation, vol. 7, pp. 2-2, 2018.

[23] L. Bermudez and M. Piasecki, "Hydrologic ontology for the web," AGUFM, vol. 2003, pp. U41B-0016, 2003.

[24] A. Algergawy, S. Babalou, F. Klan, and B. König-Ries, "Ontology Modularization with OAPTp," Journal on Data Seman-tics, vol. 9, pp. 53-83-53-83, 2020.

[25] Z. Wu, Y. Shen, H. Wang, and M. Wu, "An ontologybased framework for heterogeneous data management and its application for urban flood disasters," Earth Science Informatics, pp. 1-14, 2020.

[26] Q. Liu, Q. Bai, C. Kloppers, P. Fitch, Q. Bai, K. Taylor, et. al., "An ontology-based knowledge management framework for a distributed water information system," Journal of Hydroinformatics, vol. 15, pp. 1169-1188, 2013.

[27] C. Dolbear, J. Goodwin, H. Mizen, and J. Ritchie, "Semantic inter-operability between topographic data and a flood defence ontology," Southampton, UK: Ordnance Survey Research \& Innovation, 2005.

[28] L. Elmhadhbi, M. H. Karray, and B. Archimède, "A modular ontology for semantically enhanced inter-operability in operational disaster response," in 16th International Conference on Information Systems for Crisis Response and Management-ISCRAM, pp. 1021-1029, 2019.

[29] Z. A. S. Muhammad Hussain Mughal, "WaterOnto: Ontology of Context Aware Grid-Based Riverine Water Management System," Sukkur IBA Journal of Computing and Mathematical Sciences, vol. 1, pp. 1-12, Jan-June 20172017.

[30] M. H. Mughal, Z. A. Shaikh, and J. A. Shamsi, "WaterGrid: A wireless sensor grid for riverine water management," in Multi-Topic Conference (INMIC), 2014 IEEE 17th International, pp. 287-292, 2014.

[31] E. Yildirim and I. Demir, "An Integrated Flood Risk Assessment and Mitigation Framework: Middle Cedar River Basin Case Study," 2020.

[32] S. Yi and Y. Sun, "Upper level ontology and integration assessment modeling in digital watershed," in 2013 21st International Conference on Geoinformatics, pp. 1-6. 2013. 
[33] M. Krötzsch, F. Simancik, and I. Horrocks, "Description logics," IEEE Intelligent Systems, vol. 29, pp. 12-19, 2013.

[34] T. Rattanasawad, K. R. Saikaew, M. Buranarach, and T. Supnithi, "A review and comparison of rule languages and rule-based inference engines for the Semantic Web," in 2013 International Computer Science and Engineering Conference (ICSEC), pp. 1-6, 2013.

[35] M. Kifer, "Rule interchange format: The framework," in International Conference on Web Reasoning and Rule Systems, pp. 1-11, 2008.

[36] D. T. T. My, H. N. B. Phuong, T. T. Huong, and B. T. M. Tu, "Design of a Four-Element Array Antenna for 5G Cellular Wireless Networks," Engineering, Technology \& Applied Science Research, vol. 10, pp. 6259-6263, 2020.

[37] S. Asghar, D. Alahakoon, and L. Churilov, "A comprehensive conceptual model for disaster management," Journal of Humanitarian Assistance, vol. 1360, pp. 1-15, 2006.

[38] D. Fernandes and J. Bernardino, "Graph Databases Comparison: AllegroGraph, ArangoDB, InfiniteGraph, Neo4J, and OrientDB," in DATA, pp. 373-380, 2018.

[39] Y. Sermet and I. Demir, "An intelligent system on knowledge generation and communication about flooding," Environmental modelling \& software, vol. 108, pp. 51-60, 2018.

[40] A. Matsokis, H. M. Karray, B. Chebel-Morello, and D. Kiritsis, "An ontology-based model for providing semantic maintenance," IFAC Proceedings Volumes, vol. 43, pp. 12$17,2010$.

[41] D. De Wrachien, J. Garrido, S. T. E. F. A. N. O. Mambretti, and I. Requena, "Ontology for flood management: a proposal," WIT Transactions on Ecology and the Environment, vol. 159, pp. 3-13, 2012.

[42] P. Shvaiko and J. Euzenat, "Ontology matching: state of the art and future challenges," IEEE Transactions on knowledge and data engineering, vol. 25, pp. 158-176, 2011.

[43] J. Garrido, I. Requena, and S. Mambretti, "Semantic model for flood management," Journal of Hydroinformatics, vol. 14, pp. 918-936, 2012. 